\title{
CONTRIBUTION TO THE GEOCHRONOLOGY OF THE PERMO-TRIASSIC ALKALINE MAGMATISM OF THE ALTO PARAGUAY PROVINCE
}

\author{
VICTOR FERNANDEZ VELAZQUEZ*, CELSO DE BARROS GOMES**, WILSON TEIXEIRA**, PIERO \\ COMIN-CHIARAMONTI***
}

\begin{abstract}
RESUMO CONTRIBUIÇÃO À GEOCRONOLOGIA DO MAGMATISMO PERMO-TRIÁSSICO DA PROVÍNCIA ALTO PARAGUÁI A província alcalina do Alto Paraguai consiste em vários complexos circulares, caracterizados por rochas intrusivas capeadas por derrames de lavas e ignimbritos. As rochas são principalmente nefelina sienitos e sienitos e seus equivalentes extrusivos. Duas suítes são aparentes a partir de dados petroquímicos e isotópicos $\left({ }^{87} \mathrm{Sr} /{ }^{86} \mathrm{Sr}\right)$ : uma agpaítica predomina no norte enquanto uma miaskítica é dominante no sul. Uma tentativa de avaliar a idade da intrusão dos complexos foi feita com o emprego dos métodos K/Ar, $\mathrm{Ar} / \mathrm{Ar}$ e $\mathrm{Rb} / \mathrm{Sr}$ em rocha total e concentrados de minerais (anfibólio, feldspato alcalino e biotita).

As idades $\mathrm{K} / \mathrm{Ar}$ e Ar/Ar das fases analisadas sofreram desequilíbrios mineralógicos/geoquímicos substanciais. As idades obtidas a partir da biotita parecem mais representativas de intrusões que ocorreram entre 244 e $263 \mathrm{Ma}$. A sistemática de $\mathrm{Rb} / \mathrm{Sr}$ destaca um caráter não contaminado da(s) fonte(s) $\left({ }^{87} \mathrm{Sr}^{86} \mathrm{Sr}_{\text {initial }}=0.7037\right)$. O melhor ajuste isocrônico das amostras analisadas mostra um intervalo de idade situado entre $251 \mathrm{Ma}$ (Cerro Boggiani, Fecho dos Morros e Cerrito, área norte) e $255 \mathrm{Ma}$ (Cerro Siete Cabezas, área sul). No todo, o(s) evento(s) magmático(s) parecem estar bem situados na transição do Permiano ao Triássico.

Palavras-chaves: Paraguai Oriental, rochas alcalinas, geocronologia

ABSTRACT The alkaline province of Alto Paraguay consists of several major circular complexes, characterized by intrusive rock-types topped by lava flows and ignimbrites. The rock-types are mainly nepheline syenites and syenites and effusive equivalents; two main evolved suites are apparent from petrochemical and isotopic $\left({ }^{87} \mathrm{Si} /{ }^{86} \mathrm{Sr}\right)$ data: an agpaitic suite prevails in the northern area, whereas a miaskitic one is dominant in the south. An attempt to constrain the emplacement age of the alkaline complexes was made by $\mathrm{K} / \mathrm{Ar}, \mathrm{Ar} / \mathrm{Ar}$ and $\mathrm{Rb} / \mathrm{Sr}$ methods on whole rocks and mineral concentrates (amphibole, alkali feldspar and biotite).

$\mathrm{K} / \mathrm{Ar}$ and $\mathrm{Ar} / \mathrm{Ar}$ ages suffer of substantial mineralogical/geochemical disequilibria relative to the analyzed phases.

Dates obtained from biotite appear more suitably representative of an emplacemente age between 244 and $263 \mathrm{Ma}$. The $\mathrm{Rb} / \mathrm{Sr}$ systematic highlights an "uncontaminated" character of the source $(\mathrm{s})\left({ }^{87} \mathrm{Sr} /{ }^{86} \mathrm{Sr}_{\text {initial }}=0.7037\right) \mathrm{The}$ isochronic best fit relative to the analyzed samples shows an age span from $251 \mathrm{Ma}$ (Cerro Boggiani, Fecho dos Morros and Cerrito, northern area) to $255 \mathrm{Ma}$ (Cerro Siete Cabezas, southern area). On the whole, the magmatic event(s) seem well constrained to the Permian-Triassic transition.
\end{abstract}

Keywords: Eastern Paraguay, alkaline rocks, geochronology

INTRODUCTION The sodic-alkaline province of Alto Paraguay close to the border of Brazil (Mato Grosso do Sul) and Paraguay (Gomes et al. 1996) consists of six major, nearly circular complexes, i.e. Cerro Boggiani, Pão de Açúcar, Fecho dos Morros, Cerrito, Pedreira and Cerro Siete Cabezas and of several minor occurrences (Fig. 1). The alkaline outcrops are restricted to a narrow N-S trending lineament that parallels the Paraguay river.

The country rocks are constituted by alluvial sediments of the Paraguay river covering the Precambrian basement. In fact, widespread granitic/gneissic rocks and rhyolitic flows are known in the area as the "Rio Apa crystalline complex", with ages ranging from about 1.2 to $1.7 \mathrm{Ga}$ (Araújo et al. 1982, Amaral 1984).

The alkaline complexes are sodic intrusive, mainly nepheline syenites and syenites, topped by lava flows and ignimbrites of phonolitic affinity. From a petrochemical point of view, two main suites are apparent (Fig. 2). An agpaitic, strongly undersaturated suite is dominant in the Cerro Boggiani, Pão de Açúcar and Cerrito complexes, whereas a suite tendentiously miaskitic and oversaturated prevails in the Cerro Siete Cabezas complex. The agpaitic rocks are typically characterized by sodalite, aegirine and arfvedsonite/magnesio-arfvedsonite [range of alkaline amphiboles, $\mathrm{Fe}^{3+} /\left(\mathrm{Fe}^{3+}+\mathrm{Al}^{\mathrm{IV}}\right)$ and $\mathrm{Mg} /\left(\mathrm{Mg}+\mathrm{Fe}^{2+}\right)$, a.f.u. ratios: 0.64-0.99 and $0.48-0.70$, respectively, both in the whole population and in single samples] and the miaskitic rocks by aegirine-augite and/or katophorite/ferro-richterite/riebeckite [range of sodic- calcic amphiboles, $\mathrm{Si}$ and $\mathrm{Mg} /\left(\mathrm{Mg}+\mathrm{Fe}^{2+}\right)$, a.f.u.: $7.25-7.75$ and 0.07-0.45. respectively; range of alkaline amphiboles, $\mathrm{Fe}^{3+} /\left(\mathrm{Fe}^{3+}+\mathrm{Ar}\right)$ and $\mathrm{Mg} /\left(\mathrm{Mg}+\mathrm{Fe}^{2+}\right)$, a.f.u. ratios: 0.93-0.99 and 0.07-0.18, respectively]. Exsolved anorthoclase, biotite $\left(\mathrm{R}^{3+}=24-33 ; \mathrm{Fe}^{2+}+\mathrm{Mn}=45-61 ; \mathrm{Mg}=8-25\right.$ a.f.u. $)$, opaques, nepheline (undersaturated rock-types) or quartz (oversaturated rock-types) are ubiquitous. Common accessory minerals are apatite, sphene, fluorite and zircon. Exotic phases (e.g. astrophyllite, rosembuschite, pyrochlore) and unidentified minerals may be present.

Amaral et al. (1967) quoted a preferred age of $243 \mathrm{Ma}$ (Early-Middle Triassic; see Table 1) for the Pão de Açúcar complex based on two K-Ar biotite dates; two additional dates on K-feldspars yielded younger results in the range of 209-211 Ma. Comte \& Hasui (1971) provided a K-Ar whole rock age of $219 \mathrm{Ma}$ for the same complex (Late Triassic-Early Jurassic). More recently, Gomes et al. (1996) inferred an age between about 240 to $250 \mathrm{Ma}$ (Late Permian-Early Triassic) for the whole sodic magmatism from Alto Paraguay.

This paper is a first attempt to evaluate the timing of the magmatic event(s) affecting the northwestern fringe of the Paraná Basin by using different methods of dating, i.e. K/Ar, $\mathrm{Ar} / \mathrm{Ar}$ and $\mathrm{Rb} / \mathrm{Sr}$.

GEOCHRONOLOGY: K/Ar The analytical data are reported in Table 1, along with some recalculated data from previous work (Amaral et al. 1967, Comte \& Hasui 1971). Some samples were measured both at the "Centro de Pes-

\footnotetext{
* Curso de Pós-Graduação, Instituto de Geociências, Universidade de São Paulo, Caixa Postal 11.348, Cep 05422-970 São Paulo, Brazil

** Instituto de Geociências, Universidade de São Paulo, Caixa Postal 11.348, Cep 05422-970 São Paulo, Brazil

*** Dipartimento di Ingegneria Chimica, dell'Ambiente e delle Materie Prime, Universita di Trieste, Piazzale Europa 1, 34127 Trieste, Italy
} 


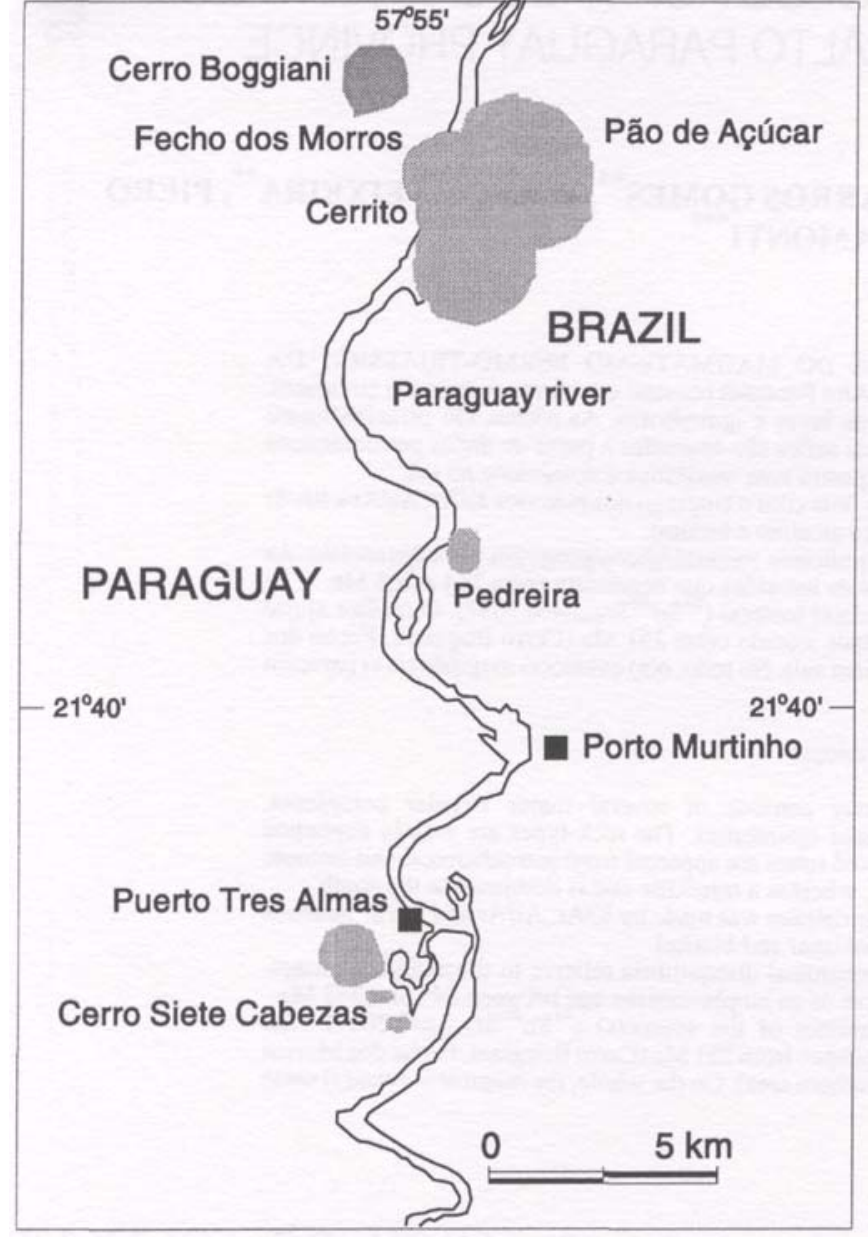

Figure l-Sketch map showing the main alkaline rock occurrences of northern Paraguay.

Figura 1 - Mapa esquemático mostrando as principais ocorrências de rochas alcalinas do Paraguai setentrional.

quisas Geocronológicas" (University of São Paulo, Brazil) and at the "Istituto di Geocronologia e Geochimica"-CNR (Pisa, Italy). Minerals were separated by conventional and gravimetric techniques. Procedures for K/Ar method in Brazil and Italy are described in Amaral et al (1967) and Del Moro et al (1982), respectively.

The whole ages range from 209 to 263 Ma. Notably, the younger dates are from alkali feldspar (211-209 Ma) and from a phonolite (219 Ma) (cf. Amaral et al. 7967, and Comte \& Hasui 1971, respectively).

The amphibole shows characteristic chemical disequilibrium that led to Ar loss, as supported by their younger dates as compared to the biotites (see Table 1). In addition, taking into account that the prevailing feldspar is anorthoclase, which has been affected by strong perthitic exsolution and secondary kaolinitization, and that whole rocks (phonolites) sometimes show hyaline texture with glass and clay material, the most reliable K-Ar data must be considered those obtained from the biotite samples. Nevertheless, duplicate analyses on biotite were performed in the Pão de Açúcar (RP-76), Cerrito (RP80), Porto Conceição (RP-9) and Cerro Siete Cabezas (RP-64) complex in order to achieve acceptable analytical errors for the K-Ar system.

$\mathrm{Ar} / \mathrm{Ar}{ }^{40} \mathrm{Ar} /{ }^{39} \mathrm{Ar}$ analyses were done on biotite (RP-100, trachyphonolite, Pão de Açúcar) and on amphibole (RP-61,

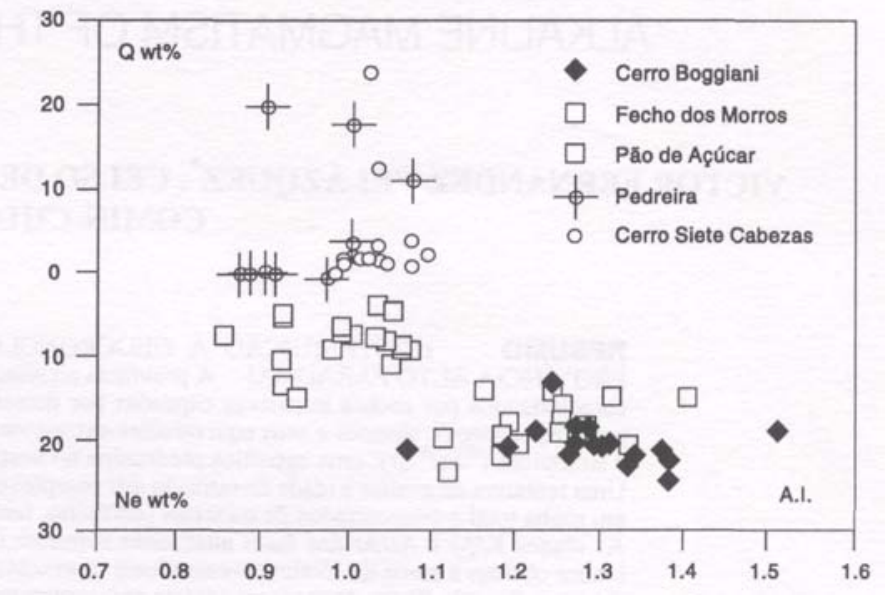

Figure 2 - Agpaitic index [A.I. $=\left(\mathrm{Na}_{2} \mathrm{O}+\mathrm{K}_{2} \mathrm{O}\right) / \mathrm{Al}_{2} \mathrm{O}_{3}$, molar ratio] vs normative quartz $(Q)$ and nepheline (Ne) of the sodic rock-types from the Alto Paraguay complexes. Chemical analyses $(N=102$, major and trace elements) are in the C.C. file and are available on request.

Figure 2 - índice agpaítico [I.A. $=\left(\mathrm{Na}_{2} \mathrm{O}+\mathrm{K}_{2} \mathrm{O}\right) / \mathrm{Al}_{2} \mathrm{O}_{3}$, proporções molares] vs quartzo normativo $(\mathrm{Q})$ e nefelina $(\mathrm{Ne})$ das rochas sódicas dos complexos alcalinos da Província Alto Paraguai. Análises químicas $(\mathrm{N}=102$, elementos maiores e traços) estão em arquivo C.C. e disponíveis mediante solicitação.

nepheline syenite, Cerro Siete Cabezas), following the procedure described in Laurenzi \& Villa (1987), using MMhb-1 as an age monitor (520 $\pm 1.7 \mathrm{Ma}$, Samson \& Alexander 1987). Both samples display slightly disturbed spectra (Fig. 3), such that the possibility to get statistically acceptable isochronic and plateau ages is very poor. The RP-100 biotite spectrum is fairly flat, but the integrated age of $242 \pm 1.6 \mathrm{Ma}$ is considered as a minimum age of the intrusion (see below).

Amphibole RP-61 shows a more disturbed spectrum, as expected from its chemical disequilibrium (see above). The lower temperature step displays low $\mathrm{Ca} / \mathrm{K}$ and a young age, probably due to exsolution of a K-rich phase (K-richterite or biotite). The middle part of the spectrum has fairly constant $\mathrm{Ca} / \mathrm{K}$, but the corresponding ages are not. The last part displays very high $\mathrm{Ca} / \mathrm{K}$ likely due to the presence of a Ca-rich phase (Gomes et al. 7996). The integrated date of $236 \pm 1.6 \mathrm{Ma}$ is a minimum age, roughly corresponding to those obtained for amphiboles from Cerro Siete Cabezas by the K/Ar method (228-230 Ma, cf. Table 1), and younger than those furnished by biotites from the same locality (244-253 Ma), which are considered to be more representative of the emplacement age of the complex. Therefore the Ar/Ar date confirms that the sodic amphibole from Alto Paraguay alkaline complexes cannot be considered as a suitable dating material and that biotite must be preferred.

$\mathrm{Rb} / \mathrm{Sr}$ The analytical data are reported in Table 2 along with those of Precambrian rhyolites of the Rio Apa crystalline basement from Fuerte Olimpo. Sr isotopic analyses were performed at the Centro de Pesquisas Geocronológicas, São Paulo, using a VG 354 mass multicollector spectrometer. The Sr isotopic compositions were adjusted to NBS987 $=0.71026$.

On the whole, Sr contents (ppm) and ${ }^{87} \mathrm{Sr} /{ }^{86} \mathrm{Sr}$ ratios follow three distinct hyperbolas (Fig. 4) relative to 1) Precambrian rhyolites from Fuerte Olimpo [age $1341 \pm 53 \mathrm{Ma}$, initial ${ }^{87} \mathrm{Sr} /{ }^{86} \mathrm{Sr}\left(\mathrm{Sr}_{\mathrm{i}}\right)=0.7133$, cf. inset A of Fig. 4]; 2) Na-alkaline rocks from Cerro Boggiani, Pão de Açúcar and Cerrito; 3 ) Na-alkaline rocks from Cerro Siete Cabezas. 

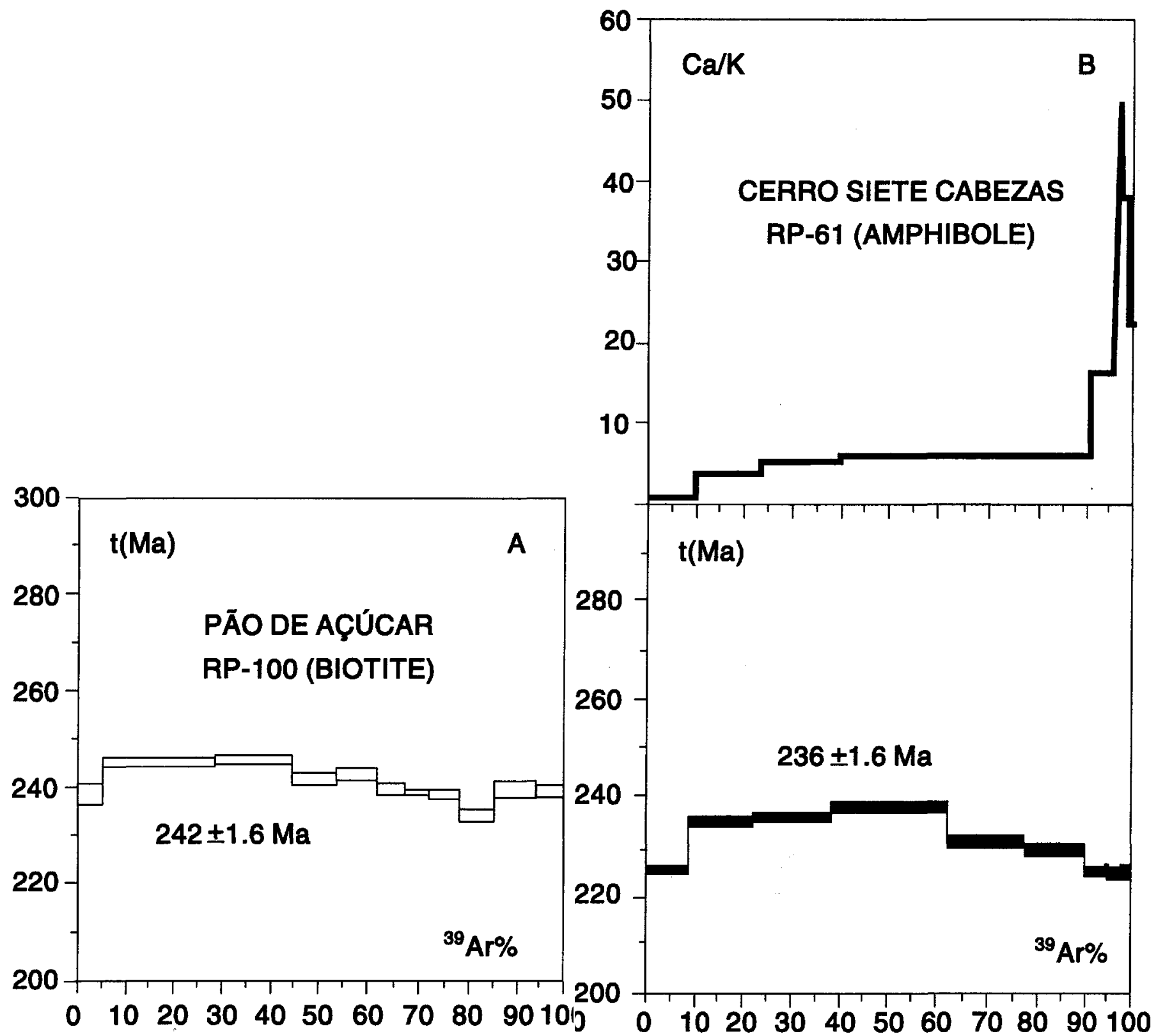

Figure 3 - Arf Ar spectra for biotite (A: RP-100, Pão de Açúcar) and amphibole (B: RP-61, Cerro Siete Cabezas) (cf. Gomes et al. 1996).

Figura 3 - Espectros ${ }^{40} \mathrm{Ar} /{ }^{39} \mathrm{Ar}$ para biotita (A: RP-100, Pão de Açúcar) e anfibólio (B: RP-61, Cerro Siete Cabezas) (cf. Gomes et al. 1996).

Notably, the two groups of alkaline rocks correspond to the agpaitic and miaskitic suites, respectively (see Fig. 2), and the mixing characteristics of the $\mathrm{Rb}-\mathrm{Sr}$ systematic may be attributed to fractionation processes from two distinct magmatic reservoirs (cf. insets B and C of Fig. 4). Moreover, the data suggest a single and rapid alkaline magmatic pulse from mantle derived magmas (initial ${ }^{87} \mathrm{Sr} /{ }^{86} \mathrm{Sr} \sim 0.703$; cf. inset B). It should be also noted that the tie line relative to the analyzed phases from RP-30 (Cerro Boggiani: alkali feldspar - whole rocks -amphibole) straddles the "alkaline" hyperbolas, due to the very low $\mathrm{Sr}$ content and to the corresponding low ${ }^{87} \mathrm{Sr} /{ }^{86} \mathrm{Sr}$ ratio of amphibole, in agreement with the previous observations about the mineralogical-geochemical disequilibrium of the sodic amphibole from Alto Paraguay alkaline complexes.
In general, the available $\mathrm{Rb}-\mathrm{Sr}$ isotopic data require caution in the dating by isochron methods both on the whole rock population (two suites) and on the mineral isochron (mineralogical and geochemical disequilibria). Some preferred solutions (best fits) are presented in Fig. 5 for the two alkaline suites, where it is apparent that the ages are well constrained at $251 \pm 2 \mathrm{Ma}$ (Cerro Boggiani, Fecho dos Morros, Cerrito) and $255 \pm 11 \mathrm{Ma}$ (Cerro Siete Cabezas) with a common $\mathrm{Sn}=$ 0.7036. This low Sn argues against appreciable effects of crust contamination. As a matter of fact, crustal contamination with basement rocks is not apparent from Fig. 4.

At any rate, the ${ }^{87} \mathrm{Sr} r{ }^{86} \mathrm{Sr}$ initial ratio, as taken from the best fit of Alto Paraguay Na-alkaline rocks (inset C of Fig. 4), along with the ${ }^{143} \mathrm{Nd} /{ }^{144} \mathrm{Nd}$ initial ratios $(0.51207$ and 0.51223, Cerro Boggiani and Fecho dos Morros, respectively, 
Table 1 - K/Ar data for representative samples of the main Na-alkaline complexes of Alto Paraguay.

Tabela 1 - Dados K/Ar de amostras representativas dos principais complexos alcalinos sódicos da Província Alto Paraguai.

\begin{tabular}{|c|c|c|c|c|c|c|}
\hline $\begin{array}{l}\text { Locality } \\
\text { Sample }\end{array}$ & Rock-type & Material & \% $\mathrm{K}$ & $\begin{array}{l}\left.{ }_{(\mathbf{A l}}^{\mathbf{A}} \mathbf{A r}_{\mathrm{rad}}\right) \\
\end{array}$ & $\begin{array}{l}\text { Ar Atm. } \\
(\%)\end{array}$ & $\begin{array}{l}\text { Age } \\
(\mathbf{M a})\end{array}$ \\
\hline $\begin{array}{l}\text { Cerro Boggiani } \\
\text { RP-27 } \\
\text { RP-30 } \\
\text { RP-43 }\end{array}$ & $\begin{array}{l}\text { Nephelinic syenite } \\
\text { Nephelinic syenite } \\
\text { Peralkal. phonolite }\end{array}$ & $\begin{array}{l}\text { Amphibole } \\
\text { Amphibole } \\
\text { Whole rock }\end{array}$ & $\begin{array}{l}1.03 \\
0.27 \\
3.50\end{array}$ & $\begin{array}{r}10.02 \\
2.62 \\
34.40\end{array}$ & $\begin{array}{r}24.42 \\
31.48 \\
8.44\end{array}$ & $\begin{array}{l}234.6 \pm 13.7 \\
234.0 \pm 9.0 \\
236.7 \pm 10.9\end{array}$ \\
\hline $\begin{array}{l}\text { Pão de Afúcar } \\
\text { RP-76 } \\
\text { RP-76* } \\
\text { RP-77 } \\
\text { RP-114 } \\
\text { RP-114* } \\
\text { SPK-1475** } \\
\text { SPK-155*** } \\
\text { SPK-098*** } \\
\text { SPK-100*** } \\
\text { SPK-156*** }\end{array}$ & $\begin{array}{l}\text { Nepheline syenite } \\
\text { Nepheline syenite } \\
\text { Nepheline syenite } \\
\text { Nepheline syenite } \\
\text { Nepheline syenite } \\
\text { Phonolite } \\
\text { Nepheline syenite } \\
\text { Nepheline syenite } \\
\text { Nepheline syenite } \\
\text { Nepheline syenite }\end{array}$ & $\begin{array}{l}\text { Biotite } \\
\text { Biotite } \\
\text { Amphibole } \\
\text { Biotite } \\
\text { Biotite } \\
\text { Whole rock } \\
\text { Biotite } \\
\text { Biotite } \\
\text { Alkali feldspar } \\
\text { Alkali feldspar }\end{array}$ & $\begin{array}{l}6.87 \\
7.70 \\
1.42 \\
6.90 \\
7.38 \\
4.72 \\
7.46 \\
7.54 \\
5.71 \\
5.68\end{array}$ & $\begin{array}{l}71.06 \\
82.49 \\
13.75 \\
71.32 \\
77.02 \\
4.17 \\
75.96 \\
75.60 \\
49.75 \\
49.07\end{array}$ & $\begin{array}{c}12.97 \\
5.0 \\
28.26 \\
7.96 \\
4.0 \\
7.0 \\
3.1 \\
3.8 \\
11.2 \\
12.0\end{array}$ & $\begin{array}{l}{[248.4 \pm 10.7]} \\
256 \pm 3 \\
233.2 \pm 7.2 \\
248.3 \pm 5.3 \\
250 \pm 3 \\
219.1 \pm 13.3 \\
244.6 \\
241.1 \\
211.3 \\
209.6\end{array}$ \\
\hline $\begin{array}{l}\text { Fecho dos Morros } \\
\text { RP-91 }\end{array}$ & Nephelinic syenite & Amphibole & 1.02 & 8.95 & 66.39 & $212.8 \pm 14.8$ \\
\hline $\begin{array}{l}\text { Cerrito } \\
\text { RP-80 } \\
\text { RP-80* }\end{array}$ & $\begin{array}{l}\text { Nephelinic syenite } \\
\text { Nephelinic syenite }\end{array}$ & $\begin{array}{l}\text { Biotite } \\
\text { Biotite }\end{array}$ & $\begin{array}{l}7.45 \\
7.88\end{array}$ & $\begin{array}{l}78.63 \\
85.53\end{array}$ & $\begin{array}{c}10.64 \\
5.0\end{array}$ & $\begin{array}{l}{[253.2 \pm 9.2]} \\
254 \pm 4\end{array}$ \\
\hline $\begin{array}{l}\text { Porto Conceifão } \\
\text { RP-9 } \\
\text { RP-9* }\end{array}$ & $\begin{array}{l}\text { Syenite } \\
\text { Syenite }\end{array}$ & $\begin{array}{l}\text { Biotite } \\
\text { Biotite }\end{array}$ & $\begin{array}{l}6.93 \\
6.83\end{array}$ & $\begin{array}{l}75.71 \\
71.79\end{array}$ & $\begin{array}{c}13.20 \\
5.0\end{array}$ & $\begin{array}{l}{[263.2 \pm 23.1]} \\
252 \pm 3\end{array}$ \\
\hline $\begin{array}{l}\text { Cerro Siete Cabezas } \\
\text { RP-61 } \\
\text { RP-64 } \\
\text { RP-64* } \\
\text { RP-69 } \\
\text { RP-70 }\end{array}$ & $\begin{array}{l}\text { Nepheline syenite } \\
\text { Nepheline syenite } \\
\text { Nepheline syenite } \\
\text { Syenite } \\
\text { Syenite }\end{array}$ & $\begin{array}{l}\text { Amphibole } \\
\text { Biotite } \\
\text { Biotite } \\
\text { Amphibole } \\
\text { Biotite } \\
\end{array}$ & $\begin{array}{l}1.74 \\
6.76 \\
7.51 \\
1.86 \\
6.10\end{array}$ & $\begin{array}{l}16.40 \\
71.39 \\
77.94 \\
17.63 \\
61.95 \\
\end{array}$ & $\begin{array}{c}34.44 \\
14.24 \\
4.0 \\
26.70 \\
19.84 \\
\end{array}$ & $\begin{array}{l}227.9 \pm 7.8 \\
{[253.4 \pm 12.5]} \\
249 \pm 3 \\
229.8 \pm 8.3 \\
244.4 \pm 10.4\end{array}$ \\
\hline
\end{tabular}

(*) "Istituto di Geocronologia e Geochimica Isotopica del CNR", Pisa, Italy; (**) Comte \& Hasui (1971); ***) Amaral et al. (1967): recalculated according to constants reported in Steiger \& Jager (1977). Results in brackets are considered as dubious (see text for details).

Table 2 - Rb-Sr isotopic data (Alto Paraguay alkaline rocks and Precambrian rhyolites). Analytical uncertainty of the Rb/Sr and $\mathrm{Sr} / \mathrm{Sr}$ isotopic ratios is shown in parentheses in last figure.

Tabela 2 - Dados isotópicos $\mathrm{Rb}$-Sr (rochas alcalinas da Província Alto Paraguai e riólitos pré-cambrianos). Incerteza analítica das razões isotópicas $\mathrm{Rb} / \mathrm{Sr}$ e $\mathrm{Sr} / \mathrm{Sr}$ está entre parênteses.

\begin{tabular}{|c|c|c|c|c|c|c|}
\hline Locality Sample & Rock-Type & Material & $\mathbf{R b}$ & $\mathbf{S r}$ & ${ }^{87} \mathrm{Rb} /{ }^{86} \mathrm{Sr}$ & ${ }^{87} \mathrm{Sr} /{ }^{86} \mathrm{Sr}$ \\
\hline $\begin{array}{l}\text { Cerro Boggiani } \\
\text { RP-27 } \\
\text { RP-29 } \\
\text { RP-30 } \\
\text { RP-30 } \\
\text { RP-30 } \\
\text { RP-33 } \\
\text { RP-35 } \\
\text { RP-39 } \\
\text { RP-40 }\end{array}$ & $\begin{array}{l}\text { Nephelinic syenite } \\
\text { Nephelinic syenite } \\
\text { Nephelinic syenite } \\
\text { Nephelinic syenite } \\
\text { Nephelinic syenite } \\
\text { Peralkal. Phonolite } \\
\text { Nephelinic syenite } \\
\text { Nephelinic syenite } \\
\text { Peralkal. Phonolite }\end{array}$ & $\begin{array}{l}\text { WR } \\
\text { WR } \\
\text { WR } \\
\text { AF } \\
\text { Amp } \\
\text { WR } \\
\text { WR } \\
\text { WR } \\
\text { WR }\end{array}$ & $\begin{array}{l}272.3 \\
341.9 \\
286.1 \\
365.6 \\
26.9 \\
344.3 \\
298.4 \\
284.7 \\
340.0\end{array}$ & $\begin{array}{r}73.1 \\
242.7 \\
95.1 \\
97.6 \\
68.8 \\
152.9 \\
120.2 \\
101.1 \\
180.0\end{array}$ & $\begin{array}{r}10.82(30) \\
4.08(11) \\
8.73(25) \\
10.88(31) \\
1.13(2) \\
6.53(18) \\
7.20(20) \\
8.17(23) \\
5.47(16)\end{array}$ & $\begin{array}{l}0.74463(6) \\
0.71625(8) \\
0.72919 \text { (9) } \\
0.74070 \text { (6) } \\
0.70949 \text { (9) } \\
0.72706 \text { (7) } \\
0.73069(9) \\
0.73680(16) \\
0.72082(7)\end{array}$ \\
\hline $\begin{array}{l}\text { Fecho dos Morros } \\
\text { RP-89 } \\
\text { RP-89 } \\
\text { RP-89 } \\
\text { RP-91* } \\
\text { RP-95 }\end{array}$ & $\begin{array}{l}\text { Nephelinic syenite } \\
\text { Nephelinic syenite } \\
\text { Nephelinic syenite } \\
\text { Nephelinic syenite } \\
\text { Nephelinic syenite }\end{array}$ & $\begin{array}{l}\text { WR } \\
\mathrm{Bi} \\
\mathrm{AF} \\
\text { WR } \\
\text { WR }\end{array}$ & $\begin{array}{r}97.8 \\
360.7 \\
46.5 \\
156.0 \\
154.5\end{array}$ & $\begin{array}{r}1126.3 \\
85.2 \\
805.7 \\
741.0 \\
641.7\end{array}$ & $\begin{array}{l}0.25(4) \\
12.30(35) \\
0.17(1) \\
0.61(1) \\
0.70(1)\end{array}$ & $\begin{array}{l}0.70449(6) \\
0.74391 \text { (8) } \\
0.70451 \text { (9) } \\
0.70562(1) \\
0.70584(10)\end{array}$ \\
\hline $\begin{array}{l}\text { Cerrito } \\
\text { RP-80 } \\
\text { RP-80 } \\
\text { RP-80 } \\
\text { RP-87 }\end{array}$ & $\begin{array}{l}\text { Nephelinic syenite } \\
\text { Nephelinic syenite } \\
\text { Nephelinic syenite } \\
\text { Nepheline syenite }\end{array}$ & $\begin{array}{l}\text { WR } \\
\mathrm{AF} \\
\mathrm{Bi} \\
\mathrm{WR}\end{array}$ & $\begin{array}{r}105.5 \\
84.4 \\
604.4 \\
119.7\end{array}$ & $\begin{array}{r}1613.0 \\
2503.0 \\
84.6 \\
813.2\end{array}$ & $\begin{array}{c}0.19(1) \\
0.10(1) \\
20.80(29) \\
0.43(1)\end{array}$ & $\begin{array}{l}0.70419(7) \\
0.70406(9) \\
0.77523(8) \\
0.70515(9)\end{array}$ \\
\hline $\begin{array}{l}\text { Cerro Siete Cabezas } \\
\text { RP-66 } \\
\text { RP-70 } \\
\text { RP-70 } \\
\text { RP-74 } \\
\text { SAT-1 RP-44 } \\
\text { SAT-1 RP-45 } \\
\text { SAT-1 RP-47 } \\
\text { SAT II RP-54 } \\
\text { SAT II RP-55 } \\
\text { SAT II RP-56 }\end{array}$ & $\begin{array}{l}\text { Syenite } \\
\text { Syenite } \\
\text { Syenite } \\
\text { Syenite } \\
\text { Syenite } \\
\text { Quartz syenite } \\
\text { Quartz syenite } \\
\text { Syenite } \\
\text { Syenite } \\
\text { Syenite }\end{array}$ & $\begin{array}{l}\text { WR } \\
\text { WR } \\
\text { AF } \\
\text { WR } \\
\text { WR } \\
\text { WR } \\
\text { WR } \\
\text { WR } \\
\text { WR } \\
\text { WR }\end{array}$ & $\begin{array}{r}80.0 \\
83.0 \\
62.1 \\
155.0 \\
211.6 \\
186.6 \\
153.1 \\
133.3 \\
154.4 \\
170.1\end{array}$ & $\begin{array}{l}252 \\
335 \\
406.2 \\
278 \\
109.8 \\
22.9 \\
82.9 \\
43.2 \\
96.9 \\
20.6\end{array}$ & $\begin{array}{r}0.92(3) \\
0.72(2) \\
0.44(1) \\
1.61(5) \\
5.58(16) \\
23.79(7) \\
5.36(2) \\
8.96(25) \\
4.62(13) \\
24.08(35)\end{array}$ & $\begin{array}{l}0.70714(8) \\
0.70606(6) \\
0.70542(7) \\
0.70939(7) \\
0.71985(11) \\
0.79755(7) \\
0.71622(6) \\
0.73800(12) \\
0.72099(6) \\
0.79646(9)\end{array}$ \\
\hline $\begin{array}{l}\text { Fuerte Olimpo** } \\
R P-10 \\
R P-16 \\
R P-18 \\
R P-19 \\
R P-20 \\
R P-22 \\
\end{array}$ & $\begin{array}{l}\text { Rhyolite } \\
\text { Rhyolite } \\
\text { Rhyolite } \\
\text { Rhyolite } \\
\text { Rhyolite } \\
\text { Rhyolite } \\
\end{array}$ & $\begin{array}{l}\text { WR } \\
\text { WR } \\
\text { WR } \\
\text { WR } \\
\text { WR } \\
\text { WR } \\
\end{array}$ & $\begin{array}{r}136 \\
122 \\
102 \\
94 \\
124 \\
100 \\
\end{array}$ & $\begin{array}{l}141 \\
134 \\
198 \\
238 \\
208 \\
265 \\
\end{array}$ & $\begin{array}{l}2.81(8) \\
2.65(8) \\
1.50(4) \\
1.15(3) \\
1.73(5) \\
1.09(5)\end{array}$ & $\begin{array}{l}0.76652(10) \\
0.76504(8) \\
0.74097(9) \\
0.73573(6) \\
0.74737(9) \\
0.72983(1) \\
\end{array}$ \\
\hline
\end{tabular}

*Comin-Chiaramonti et al. (1996); **Precambrian rhyolites within the basement. 


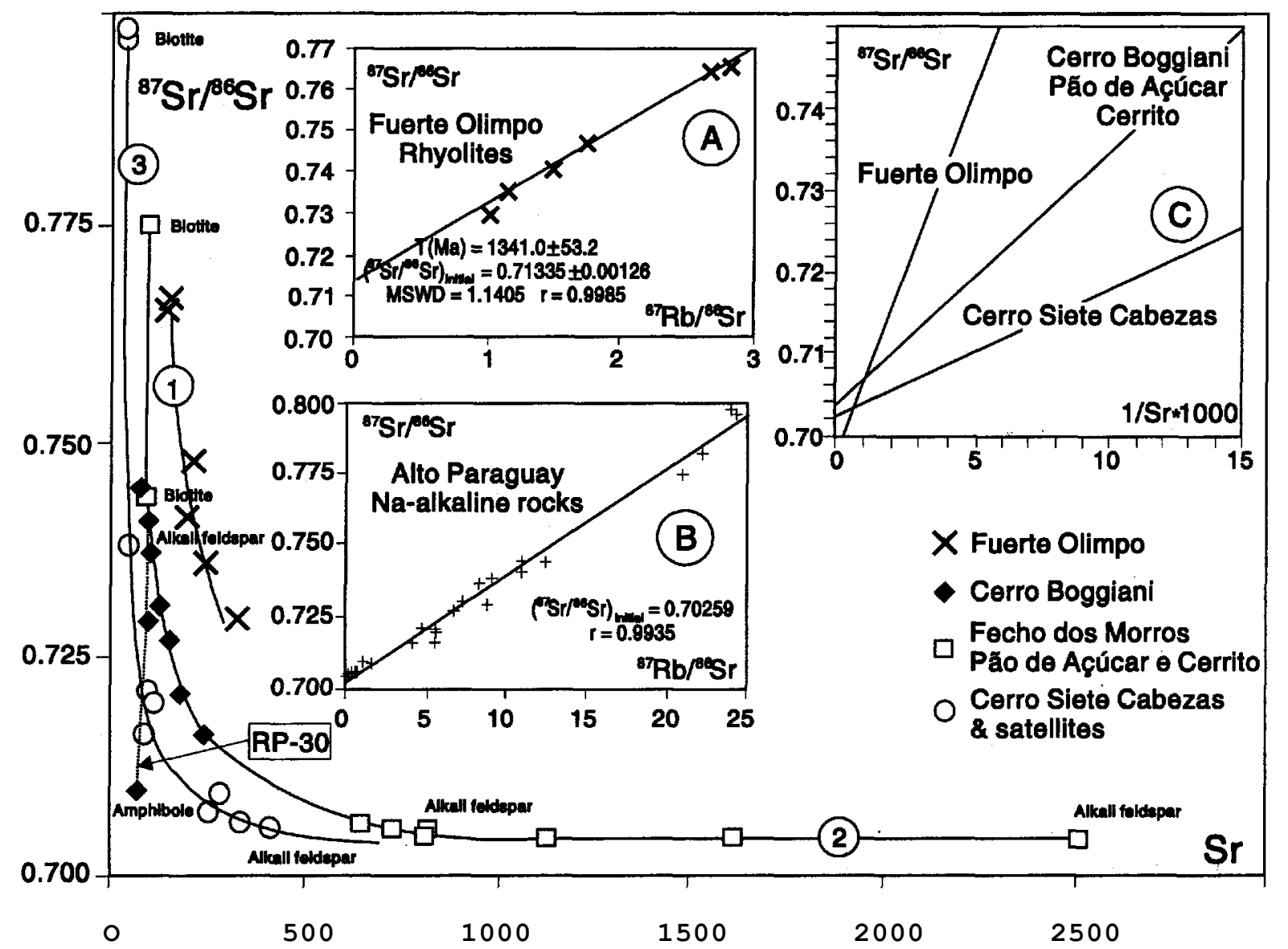

Figure 4-Sr vs ${ }^{87} \mathrm{Sr}{ }^{86} \mathrm{Sr}$ relationship s for magmatic rock-types from Alto Paraguay. Insets: A, isochron relative to Fuerte Olimpo Precambrian rhyolites; $B-10^{387} \mathrm{Sr}{ }^{86} \mathrm{Sr} v{ }^{87} \mathrm{Sr}{ }^{86} \mathrm{Sr}$; C, best fit relative to the whole Na-alkaline population.

Figura 4 - Relações Sr vs ${ }^{87} \mathrm{Sr}{ }^{86} \mathrm{Sr}$ de rochas magmáticas do Alto Paraguai. A, isócrona dos riolitos pré-cambrianos de Fuerte Olimpo; $\mathrm{B}, 10^{3} / \mathrm{Sr}$ vs ${ }^{87} \mathrm{Sr} /{ }^{86} \mathrm{Sr} ; \mathrm{C}$, melhor ajuste de toda a população de rochas alcalinas ricas em $\mathrm{Na}$.
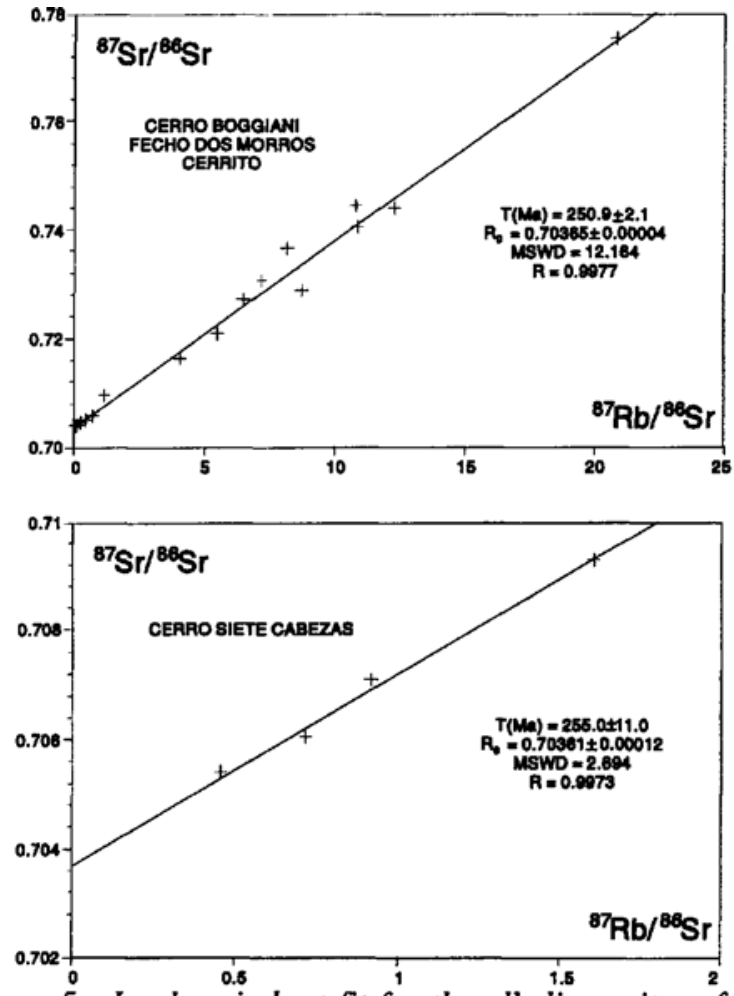

Figura 5 - Isochronic best fit for the alkaline suites of Alto Paraguay.

Figura 5 - Melhores ajustes isocrônicos para as suftes alcalinas do Alto Paraguai.
$\mathrm{T}^{\mathrm{DM}}=954.1068 \mathrm{Ma}$ : cf Comin-Chiaramonti et al 7996) point to a source having $\mathrm{Sm} / \mathrm{Nd} \leq \mathrm{CHUR}$ and a depleted $\mathrm{Rb} / \mathrm{Sr}$ ratio (contamination of mantle derived magmas with lower crust granulites?).

CONCLUDING REMARKS Two main suites of Na-alkaline rocks, agpaitic and miaskitic, respectively, are apparent for the alkaline complexes in the Alto Paraguay region near the border of Brazil (Mato Grosso do Sul) and Paraguay on the basis of petrochemical features and geochemical trends (e.g. Sr vs ${ }^{87} \mathrm{Sr} /{ }^{86} \mathrm{Sr}$ ): i.e. 1) agpaitic and/or undersaturated (Cerro Boggiani, Pão de Açúcar, Fecho dos Morros and Cerrito); 2) miaskitic and oversaturated (Cerro Siete Cabezas).

The methods of dating show isotopic complexity linked above all to mineralogical characteristics of the analyzed materials. However, the critical interpretation of the available data for both two suites points to the following notional ages:

1) $\mathrm{Rb} / \mathrm{Sr}$, best fits: $251+2 \mathrm{Ma}$ (Cerro Boggiani, Fecho, dos Morros and Cerrito) and 255 $\pm 11 \mathrm{Ma}$ (Cerro Siete Cabezas; 2) Ar/Ar: 242 $\pm 1.6 \mathrm{Ma}$ (Pão de Açúcar, minimum age); 3) K/Ar, range of acceptable "minimum ages" on biotites: 241-256 Ma (Pão de Açúcar), $252 \mathrm{Ma}$ (Porto Conceição) and 244-249 Ma (Cerro Siete Cabezas).

Although a great selection of rock-samples and of mineral phases is needed in order to minimize effects relative to the apparent disequilibria, such as reaction rims, exsolutions, hydrothermal alteration and weathering, the magmatic events of Alto Paraguay seem well constrained to the Permian-Triassic transition. Moreover the standard deviations on the age data do not support chronologically distinct magmatic pulses 
f e.g miaskitic and agpaitic magmatism), and the low initial $\mathrm{Sr} /{ }^{8} \mathrm{Sr}$ ratios favours the hypothesis of a common, isotopically homogeneous, mantle source and high rate of ascent ofthe generated magmas.

Finally, the similarity between the Alto Paraguay Province and the "uncontaminated" rock-types of the Velasco sodic-alkaline Province (eastern Bolivia; Fletcher \& BeddoeStephens 7987), emplaced into Precambrian gneiss (1366 Ma) during Late Jurassic or very Early Cretaceous (134-143 Ma, initiaP $\mathrm{Pr}^{86} \mathrm{Sr}=0.7045, \mathrm{Rb} / \mathrm{Sr}=0.16$; Darbyshire \& Fletcher $1979)$ should be stressed. Both provinces $\left(\mathrm{e}^{\mathrm{t}} \mathrm{Sr}=-7\right.$ and -10 , respectively) belong to the southwestern part of the Amazon Craton (e.g. Teixeira et al. 1989) and seem to testify to a peculiar lithospheric segment of mantle sources quite distinct from those of "potassic" affinity within the intracratonic Paraná Basin " affinity (Comin-Chiaramonti et al. 7996).

Acknowledgements The authors are deeply thanked to Dr. Marinella Laurenzi of the "Istituto di Geocronologia" (CNR), Pisa, Italy, for the valuable help in obtaining $\mathrm{Ar} / \mathrm{Ar}$ and $\mathrm{K} / \mathrm{Ar}$ age determinations. Financial support from Brazilian (FAPESP: Proc. 90/3692-7) and Italian (MURST $40 \%$ ) agencies is gratefully acknowledged. Thanks are also due to Dr. Thomas R. Fairchild for reviewing the manuscript.

\section{REFERENCES}

AMARAL, G. 1984. Província Tapajós e Rio Branco. In: ALMEIDA, F.F.M. \& HASUI, Y., eds. O Pré-Cambriano do Brasil. Editora Edgard Blücher Lida., São Paulo, p. 6-35.

AMARAL, G.; BUSHEE, J.; CORDANI, U.G.; REYNOLDS, J.H. 1967. Potassium-argon ages of alkaline rocks from Southern Brazil. Geochimica et Cosmochimica Acta, 31:117-142.

ARAÚJO, H.J.T.; SANTOS NETO, A.; TRINDADE, C.A.H.; PINTO, J.C.A.; MONTALVÃO, R.M.G.; DOURADO, T.D.C.; PALMEIRA, R.C.B.; TASSINARI, C.C.G. 1982. Folha SF 21, Campo Grande: Geologia. In: Projeto Radambrasil, MME/SG, Rio de Janeiro, 28, p. 83-84.

COMIN-CHIARAMONTI, P.; CUNDARI, A.; PICCIRILLO, E.M.; GOMES, C.B.; CASTORINA, F.; CENSI, P.; DE MIN, A.; MARZOLI, A.; SPEZIALE, S.; VELAZQUEZ, V.F. 1996. Potassic and sodic igneous rocks from Eastern Paraguay: their origin from the lithospheric mantle and genetic relationships with the associated Paraná flood tholeiites. Journal of Petrology (in press).

COMTE, D. \& HASUI, Y. 1971. Geochronology of Eastern Paraguay by the potassium-argon method. Revista Brasileira de Geociências., 1:33-43.

DARBYSHIRE, D.P.F. \& FLETCHER, C.J.N. 1979. A Mesozoic alkaline province in eastern Bolivia. Geology, 7:545-548.

DEL MORO, A.; PUXEDDU, M.; BROZZOLO R.F.; VILLA, I.M. 1982. $\mathrm{Rb} / \mathrm{Sr}$ and $\mathrm{K} / \mathrm{Ar}$ ages on minerals at temperatures of $300-400{ }^{\circ} \mathrm{C}$ from deep wells in the Larderello Geothermal Field (Italy). Contributions to Mineralogy and Petrology, 81:340-349.
FLETCHER, C.J.N. \& BEDDOE-STEPHENS, B. 1987. The petrology, chemistry and crystallization history of the Velasco alkaline province, eastern Bolivia. In: FITTON, J.C. \& UPTON, B.G.J., eds. Alkaline igneous rocks, Geological Society Special Publication, 30:403-413.

GOMES, C.B.; LAURENZI, M.A.; CENSI, P.; DE MIN, A.; VELAZQUEZ V.F.; COMIN-CHIARAMONTI, P. 1996. Alkaline magmatism from northern Paraguay (Alto Paraguay): a Permo-Triassic Province. In: COMIN-CHIARAMONTI, P. \& GOMES, C.B., eds. Alkaline magmatism in Central-Eastern Paraguay. Relationships with coeval magmatism in Brazil. EDUSP/FAPESP, São Paulo, p. 223-230.

LAURENZI, M.A. \& VILLA, I.M. (1987). ${ }^{40} \mathrm{Ar} /{ }^{39} \mathrm{Ar}$ chronostratigraphy of Viço ignimbrites. Periódico di Mineralogia, 56:285-295.

SAMSON, S.D. \& ALEXANDER Jr., E.G. 1987. Calibration of the interlaboratory ${ }^{40} \mathrm{Ar} /{ }^{39} \mathrm{Ar}$ dating standard, MMhb-1. Chemical Geology, 66:27-34.

STEIGER, R.H. \& JAGER, E. 1977. Subcommission on geochronology: Convention on te use of decay constants in geo- and cosmochronology. Earth Planetary Science Letters, 36:359-362.

TEIXEIRA, W.; TASSINARI, C.C.G.; CORDANI, U.G.; KAWASHITA, K. 1989. A review of the geochronology of the Amazonian Craton: tectonic implications. Precambrian Research, 42:213-227.

MANUSCRITO A851

Recebido em 10 de setembro de 1996 Revisão do autor em 10 de março de 1997 Revisão aceite em 12 de março de 1997 